\title{
Coexistence of psoriasis and pemphigoid - only a coincidence?
}

\author{
MACIEJ LAZARCZYK ${ }^{1,2}$, KATARZYNA WOZNIAK ${ }^{1}$, NORITO ISHII ${ }^{3}$, ANNA GORKIEWICZ-PETKOV ${ }^{1}$, \\ TAKASHI HASHIMOTO ${ }^{3}$, ROBERT SCHWARZ ${ }^{4}$ and CEZARY KOWALEWSKI ${ }^{1}$ \\ Departments of ${ }^{1}$ Dermatology, ${ }^{2}$ Histology and Embryology, Medical University of Warsaw, \\ Warsaw, Poland; ${ }^{3}$ Department of Dermatology, Kurume University School of Medicine, Kurume, Japan; \\ ${ }^{4}$ Department of Dermatology, New Jersey Medical School, Newark, NJ, USA
}

Received March 1, 2006; Accepted May 3, 2006

\begin{abstract}
It has previously been reported that pemphigoid coexists with psoriasis more frequently than it could be predicted on the basis of random distribution in the general population. In this study we present three psoriatic patients who developed tense blisters, which most likely were not provoked by anti-psoriatic treatment. Diagnosis of bullous pemphigoid in these cases was established by an overlay antigen mapping technique by laser confocal microscopy, immunoblotting and ELISA. In the context of these cases and the literature, we also discuss possible reasons for the coexistence of psoriasis and pemphigoid as well as selected aspects of diagnosis and therapy of patients simultaneously suffering from these two diseases.
\end{abstract}

\section{Introduction}

Psoriasis vulgaris is a common disease, with an approximate prevalence in Europe of $2 \%(1,2)$. On the other hand, bullous pemphigoid (BP) is uncommon, with an annual frequency of about 1 new case per 100,000 people, and is seen predominantly in middle aged and older individuals $(3,4)$. It has been reported that the true coincidence rate of these two entities is higher than it would be predicted on the basis of their random distribution in the general population (5), suggesting some kind of link between them. Nevertheless, studies on the coincidence of psoriasis and pemphigoid in the literature are still sparse; the reason of this phenomenon remains obscure. It has been found that besides bullous pemphigoid also pemphigoid with antibodies directed against autoantigen with molecular weight $\sim 200 \mathrm{kDa}$ (antip200 pemphigoid) coexists with psoriasis (6). In almost all of the reported cases, psoriasis preceded pemphigoid development. Obviously, it could be due to the lower average

Correspondence to: Dr Cezary Kowalewski, Department of Dermatology, Medical University of Warsaw, Koszykowa 82a, 02-008 Warsaw, Poland

E-mail: ckowalew@amwaw.edu.pl

Key words: pemphigoid, psoriasis, laser scanning confocal microscopy age of onset in case of psoriasis, as compared to pemphigoid. Nevertheless, that may not be the only explanation. It seems possible that some factors related to the course of psoriasis, including anti-psoriatic treatment, especially phototherapy or/and tars, may play a role.

In this study, we present three cases of bullous pemphigoid in patients suffering from psoriasis, which most likely were not triggered by anti-psoriatic treatment.

\section{Materials and methods}

\section{Clinical data}

Patient 1. A 72-year-old Caucasian woman, suffering from psoriasis vulgaris for 55 years, was well controlled using only topical steroids (hydrocortisone, flumetasone) and occasionally $5 \%$ salicylic ointment. Systemic anti-psoriatic therapy, particularly ultraviolet-based phototherapy, has never been applied. The patient was referred because of a sudden bullous eruption, which had started 4 weeks earlier. At the time of examination, the psoriatic papules were limited to the skin of lower back. Tense blisters were present at the border of intact skin and a psoriatic plaque of her lower back (Fig. 1).

Oral tetracycline (500 mg 3 times a day) and nicotinamide (400 mg twice daily) were promptly introduced. Simultaneously, topical clobetasol twice a day was added. The psoriatic lesions did not need any additional specific treatment. In a few days the blisters vanished and new ones have not reappeared.

Patient 2. An 81-year-old Caucasian man, suffering from psoriasis vulgaris for 68 years, was well controlled using only topical steroids. Neither tar- nor ultraviolet-based phototherapy has been used. The patient was referred due to a disseminated extensive bullous eruption, sparing skin of the head and neck, which started one month prior to admission. At the time of examination, the psoriatic papules were limited to the lower back, extremities and scalp.

Oral tetracycline (500 $\mathrm{mg}$ thrice a day), nicotinamide (200 mg thrice a day), clemastine (1 mg twice a day) and hydroxyzine $(25 \mathrm{mg}$ ) were expeditiously introduced. Simultaneously, topical clobetasol twice a day was added. This treatment regimen was judged ineffective, as new bullae were appearing. Thus, after two weeks, methotrexate $7.5 \mathrm{mg}$ once a week was added with clinical improvement - healing 


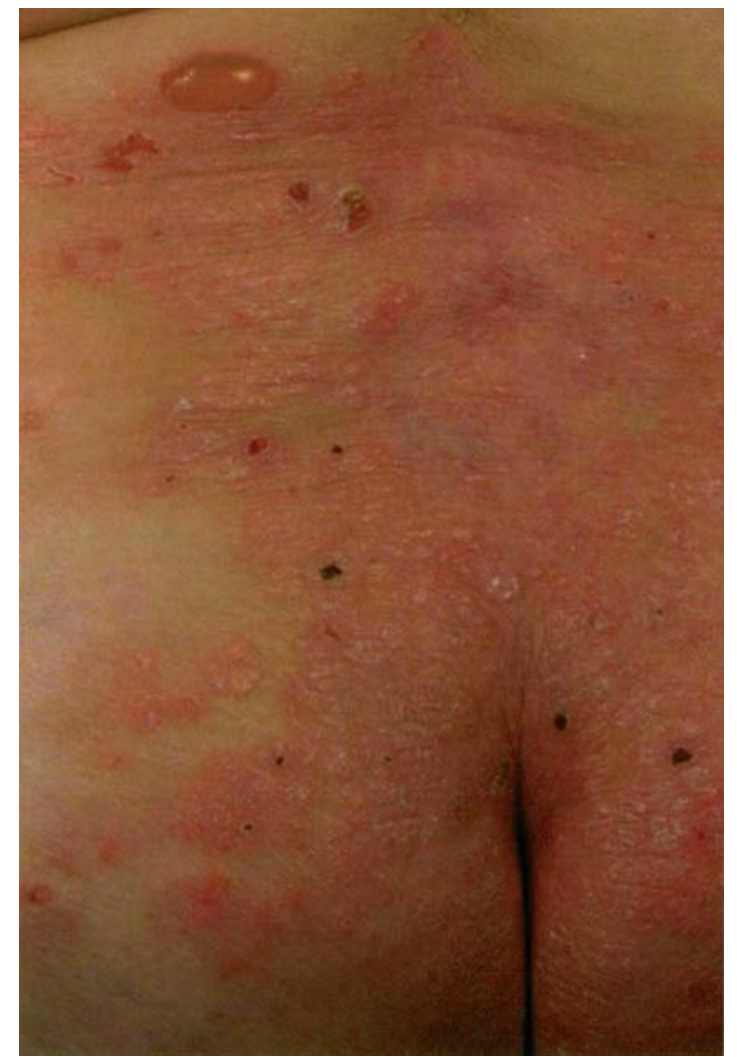

Figure 1. Patient 1 at admission to our department - a tense blister and a few erosions at the border of a psoriatic plaque localized in the lower back.

of the psoriatic plaques and complete inhibition of new blister formation.

Patient 3. A 71-year-old Caucasian man, suffering from localized psoriasis vulgaris for 20 years, with plaques limited to the knees and elbows, was well-controlled solely with topical steroids and calcipotriol. Ultraviolet-based phototherapy has never been applied in this patient. The patient was referred to our department due to a severe bullous eruption on the lower extremities, forearms and trunk, which started 3 weeks prior to admission. At the time of examination no psoriatic lesions were observed.

Oral prednisone $(0.7 \mathrm{mg} / \mathrm{kg})$, clemastine (1 mg twice a day) and topical clobetasol were promptly provided. This treatment was inefficient as new blisters continued to appear during the next 11 days. Thereafter, dapson (50 mg daily) was added to corticosteroids and continued for 9 more days. The combination of steroids and dapson was also ineffective - new blisters were continuously appearing during this period. Thus, dapson was discontinued and metotrexate $15 \mathrm{mg}$ twice a week was added. Introduction of methotrexate $(15 \mathrm{mg})$ together with prednisone $(0.7 \mathrm{mg} / \mathrm{kg})$ significantly improved treatment efficiency and controlled the disease. The preexistent blisters started healing and new ones did not appear. After 3 weeks the corticosteroid was tapered and eventually discontinued, as the patient was controlled with oral methotrexate $7.5 \mathrm{mg}$ once a week.

\section{Laboratory techniques}

Identification and localization of the in vivo-bound antibodies. Direct immunofluorescence technique on patients' skin biopsy specimens with the use of standard fluoresceinlabeled anti-human conjugates directed against a different class of human immunoglobulins and complement, was performed.

To establish the precise localization of the in vivo bound immunoglobulins within the basement membrane zone (BMZ), laser scanning confocal microscopy (LSCM) was performed, as previously described (7). Briefly, cryosected biopsy specimens, taken from perilesional skin, as well as two control biopsy specimens taken from bullous pemphigoid (positive against $180 \mathrm{kDa}$ by immunoblotting) and anti-p200 anti-p200

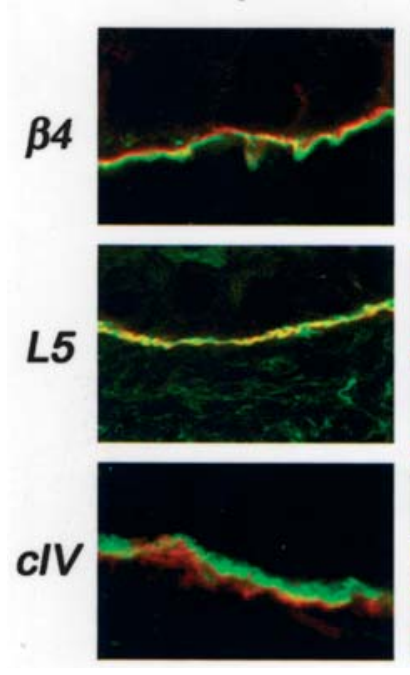

BP180
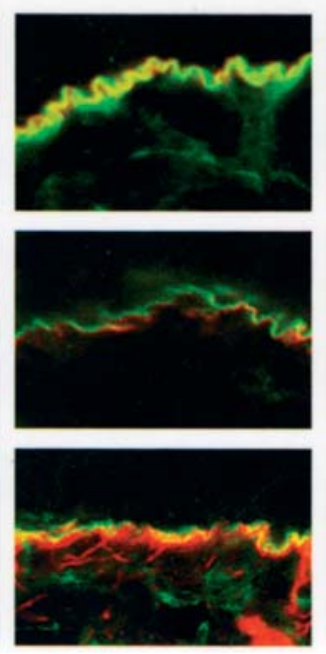

patient 1
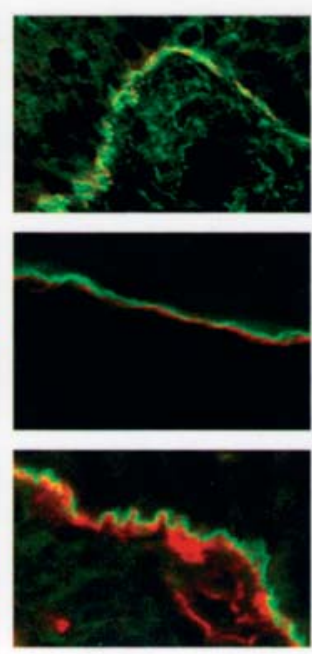

patient 2
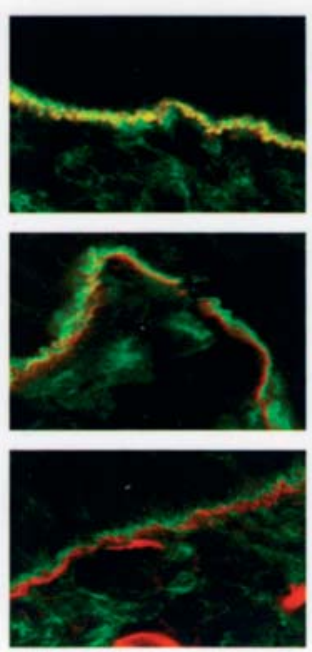

patient 3
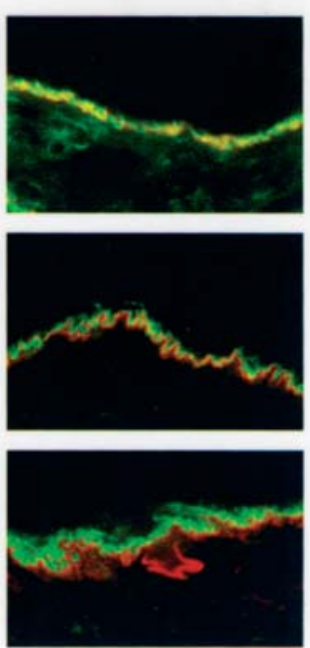

Figure 2. The overlay antigen mapping study using laser confocal microscopy shows in vivo bound IgG (stained green) in all studied patients with coexistence of psoriasis and bullous disease (patients 1-3) to be present above the collagen IV (cIV) and laminin-5 (L5) (stained red) and co-localized with ß4-integrin (ß4) (yellow color due to overlaping green and red)) similarly to control bullous pemphigoid (BP180). In a control case of anti-p200 pemphigoid (anti-p200), in vivo bound IgG are present below the localization of 34 -integrin, above collagen IV and co-localized with laminin-5. Magnification $\mathrm{x} 2500$. 


\section{BP180-NC16a}

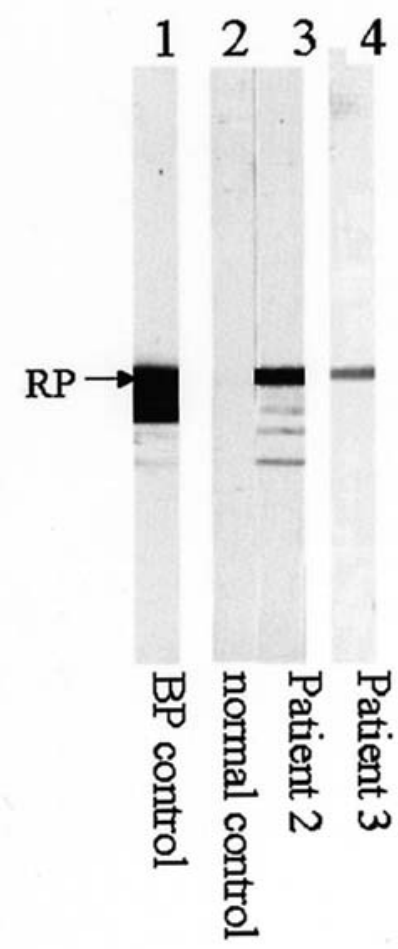

Figure 3. The results of immunoblotting using recombinant proteins of BP180 NC16a domain. The control BP serum (lane 1), patient 2 serum (lane 3) and patient 3 serum (lane 4) reacted with the recombinant protein (indicated by an arrow on the left), while normal control serum did not react with this protein (lane 2).

pemphigoid (confirmed by immunoblotting), were incubated with monoclonal antibodies directed against B4-integrin (Chemicon, Temecula, CA), laminin-5 (Chemicon, International, clone P3E4) and collagen IV (Sigma, Aldrich, Steinheim, Germany, clone COL-94). Subsequently, a mixture of rabbit anti-mouse Cy5-conjugated antibody (Chemicon, International) and FITC-conjugated goat antihuman IgG antibodies (Kappel) was added. Cryosections were viewed under LSCM (Radiance 2000 Bio-Rad). Immunofluorescence images were overlaid by an image processing system integrated in the LSCM and photographed.

Identification of the circulating antibodies. Circulating antibodies were detected by the standard indirect immunofluorescence technique, using monkey esophagus and salt split skin as substrates. Further detection of the antibodies bound to the substrate was performed analogically as in the case of direct immunofluorescence. The circulating antibodies were also further identified by the Western immunoblot technique on dermal and epidermal extracts and with the recombinant protein NC16a of BP180 antigen. Moreover, the identification of the antibody was also studied by ELISA with recombinant $\mathrm{NC16}$ a protein.

\section{Results}

Identification and localization of the in vivo-bound antibodies. In case of all studied patients, direct immuno- fluorescence performed on apparently normal patient skin showed linear IgG and C3, but not IgM or IgA, deposits along BMZ (data not shown).

The overlay antigen mapping using LSCM showed that in vivo bound $\mathrm{IgG}$ in all 3 studied patients with coexistence of psoriasis and bullous disease are localized above the collagen IV and laminin-5 and co-localized with B4-integrin similarly to control bullous pemphigoid. Conversely, in a control case of anti-p200 pemphigoid, in vivo bound IgG was found to be present below the localization of B4-integrin but above collagen IV and co-localized with laminin-5 (Fig. 2).

Identification of the circulating anti-BMZ antibodies. In patient 1 no circulating antibody was detected by immunofluorescence, immunoblotting and ELISA. In patients 2 and 3 indirect immunofluorescence on monkey esophagus revealed anti-BMZ antibodies at the 1:1280 and 1:160 titers respectively, reacted with the epidermal side of split skin. Using an immunoblotting technique the sera of patients 2 and 3 reacted with BP $180 \mathrm{kD}$ antgien in epidermal extract and with the recombinant NC16a protein of $180 \mathrm{kDa}$ antigen (Fig. 3). The ELISA technique with BP180 protein showed positive reactivity (index 20.6 in case 2 and index 22.3 in case 3 ).

\section{Discussion}

The first case of bullous eruption in psoriatic patient was reported by Bloom in 1929 (8). Despite Bloom's description of this disease as pemphigus, histological analysis suggests that it was in fact BP. Subsequently, some new cases of BP suffering from psoriasis have been described beginning from the 1970s (9-12). At present, there are $>60$ studies concerning the coexistence of psoriasis and subepidermal bullous diseases. These cases of bullous disease were usually diagnosed as BP, on the basis of histological examination and direct immunofluorescence only. However, it cannot be excluded that in some of these patients, instead of BP, other subepidermal bullous diseases existed, e.g. epidermolysis bullosa acquisita (EBA). In more recent studies, diagnosis was verified by indirect immunofluorescence in $1 \mathrm{M}$ salt-split skin test and/or immunoblotting (13-19). On the basis of these results, it has been proposed that autoimmune subepidermal bullous diseases previously considered as BP, could be divided at least into two groups: BP with autoantibodies directed against hemidesmosomal antigens $(14-16,19)$ and a novel, pemphigoid-like subepidermal bullous disease with antibodies directed against the novel antigen, localized at the border of lamina lucida and lamina densa $(6,14,17,19)$. Subsequently, it has been found that molecular weight of this new antigen is $\sim 200 \mathrm{kDa}$ $(6,17,19)$. Recently the antigen has been partially characterized (20). It is noteworthy that among the psoriatic patients pemphigoid with antibodies against $200 \mathrm{kDa}$ antigen (antip200 pemphigoid) seems to occur much more often than in the general population. However, its real prevalence is difficult to estimate, since in a great majority of patients reported in literature, appropriate analysis with immunoblotting technique has not been performed. Nevertheless, based on data recently discussed by Yasuda et al (19), one may hypothesize that $\sim 50 \%$ of pemphigoid cases in psoriatic individuals could be anti-p200 pemphigoid - a percentage 
much higher than in the general population. The reason for this remains obscure. A majority of anti-p200 pemphigoid cases in psoriatic patients were found in the Japanese population. Thus, it is tempting to speculate that development of a particular type of pemphigoid (BP or anti-p200 pemphigoid) in the psoriatic patient could be determined, at least in part, by genetic pattern. Certainly, as has been suggested (19), the higher incidence of anti-p200 pemphigoid among Japanese might result from the fact that most of the immunoblotting studies on these patients were undertaken by a Japanese group and the real prevalence of anti-p200 pemphigoid in the European population could be underestimated. However, in a majority of the more recent European studies, psoriatic patients had subepidermal bullous diseases with antibodies bound to the roof of an artificial blister in $1 \mathrm{M}$ salt-split skin test (14-16), which strongly suggests that these patients had BP, because anti-p200 pemphigoid antibodies bind predominantly to the bottom of an artificial blister $(6,17,19)$.

The cases presented herein are examples of Caucasian psoriatic patients suffering from subepidermal blistering disease, which was not drug-triggered. The results of confocal microscopy examination of all three cases excluded the diagnosis of EBA, anti-p200 pemphigoid and favored BP since immunodeposits were localized above laminin-5, at the level of 34 -integrin. It suggests that in vivo bound $\operatorname{IgG}$ was restricted to the upper part of lamina lucida, similarly to skin of control anti-180 kDa BP patient. In case of control anti-p200 pemphigoid, immunoglobulin deposits were found to be present below the localization of $\beta 4$-integrin and above collagen IV, at the level of laminin-5, which corresponds to their ultrastructural localization in the lamina lucida - lamina densa border. In the case of patients 2 and 3 the diagnosis of BP was unequivocally proven with results of Western immunoblot and ELISA analyses revealing antibodies directed against NC16a recombinant protein of $180 \mathrm{kDa}$ antigen, characteristic for BP. It needs to be emphasized, that as we demonstrated in this study, that confocal microscopy is a useful method allowing differential diagnosis of subepidermal blistering disease, especially in cases where immunoblotting or ELISA do not identify circulating antibodies. Thus, confocal microscopy may be considered as an alternative technique for electron microscopy, both a gold standard for localization of the antibodies bound to basement membrane.

Regardless of whether BP or anti-p200 pemphigoid coexists with psoriasis, the reason for this phenomenon remains obscure. Since in almost all of the reported cases psoriasis preceded pemphigoid development, it has been postulated that some factors associated with pathogenesis of psoriasis could be responsible for triggering and/or promoting bullous eruption. Presumably, 'the immune status' of psoriatic skin facilitates recognition of appropriate autoantigens in somehow predisposed humans, eventually leading to pemphigoid initiation.

However, the mechanisms of these interactions between pemphigoid and psoriasis are poorly understood and remain speculative. For instance, it is possible that skin infiltrating leukocytes and proteases released from them may participate in destruction of BMZ leading to presentation of some autoantigens. Simultaneously, psoriasis-associated inflammatory process could promote autoimmune reaction, once initiated. It has previously been found that neutrophil-derived elastase is bound to $\mathrm{BMZ}$ within psoriatic plaques and probably takes part in the destruction of epidermal-dermal junction (21). Importantly, also uninvolved psoriatic skin displays decreased anti-elastase activity (22) and might be more susceptible to proteolytic cleavage. Thus, it cannot be excluded that such conditions could facilitate access to some BMZ autoantigens. It has been reported that neutrophil elastase is able to cleave type XVII collagen (BP180 autoantigen) and to contribute to subepidermal blister formation $(23,24)$. However, increased lesional elastase activity was found also in the case of contact dermatitis and atopic dermatitis (25), the diseases that have not been found to predispose for pemphigoid development. Thus, release of proteolytic enzyme within psoriatic skin is the only one aspect of a much more complicated immuno-pathological process, and it cannot be expected that this could entirely explain the reasons of pemphigoid and psoriasis coexistence. Certainly, the coincidence of these two diseases is also determined by other, unknown immunopathological factors.

On the other hand, it has been proposed that a high rate of bullous diseases among psoriatic patients is not due to a real coincidence between psoriasis and bullous diseases, but results from anti-psoriatic therapy used. This hypothesis could be supported by the fact that there are barely any cases where appearance of blisters was not directly preceded by psoriasis treatment, excluding topical steroids $(5,26)$. Among anti-psoriatic therapeutics, phototherapy (PUVA) $(10-12,15-16,27)$ and tars $(10)$ are the most common factors suspected for triggering pemphigoid. It is in accordance with well-known observations that ultraviolet radiation is able to induce pemphigoid and to exacerbate course of bullous disease, both in healthy individuals (28) and in patients suffering from other skin disorders $(29,30)$.

In our psoriatic patients, it is difficult to point out factors that were involved in the initiation of pemphigoid. It is unlikely that they were drug-triggered pemphigoid since the only drugs used directly before blister eruption were topical steroids and/or calcipotriol, with no phototherapy or tars employed.

Regardless of the reasons of pemphigoid and psoriasis coexistence, patients simultaneously suffering from both of these diseases represent a therapeutical challenge. Treatment of pemphigoid with steroids is sometimes ineffective and may also lead to psoriasis exacerbation during their withdrawal, as in patient 2. Therefore, wherever possible, treatment with systemic steroids should be avoided. On the other hand, phototherapy as anti-psoriatic therapy is contraindicated because it could exacerbate pemphigoid. Therefore, in the cases of coexistent pemphigoid and psoriasis several different therapeutical schemes encompassing methotrexate $(10,14,15)$, dapson (31), azathioprine $(26,27,32)$, cyclosporine $(33,34)$, erythromycin with etretinate (35), acitretin (36), cyclophosphamide (11) and mycophenolate mofetil (37) have been proposed. We have demonstrated herein that therapy with metotrexate can be an effective therapeutical approach. We also show that in the case of coexistent mild to moderately severe bullous pemphigoid and psoriasis, only treatment with topical clobetasol, oral tetracyclines and nicotinamide may be adequate. Such a therapeutical approach should be 
recommended, especially for elderly patients, for whom aggressive systemic treatment is undesirable.

\section{Acknowledgements}

M. Lazarczyk is the recipient of the Stipend for Young Scientists from the Foundation for Polish Science and the Foreign Stipend for Young Doctors from the Foundation for Polish Science. This study was supported by a grant from the Polish Scientific Research Committee (No. 2PO5B 065 30).

\section{References}

1. Brandrup $\mathrm{F}$ and Green A: The prevalence of psoriasis in Denmark. Acta Derm Venereol 61: 344-346, 1981.

2. Nevitt GJ and Hutchinson PE: Psoriasis in the community: prevalence, severity and patients' beliefs and attitudes towards the disease. Br J Dermatol 135: 533-537, 1996.

3. Bernard P, Vaillant L, Labeille B, Bedane C, Arbeille B, Denoeux JP, Lorette G, Bonnetblanc JM and Prost C: Incidence and distribution of subepidermal autoimmune bullous skin diseases in three French regions. Bullous Diseases French Study Group. Arch Dermatol 131: 48-52, 1995.

4. Jung M, Kippes W, Messer G, Messer G, Zillikens D and Rzany B: Increased risk of bullous pemphigoid in male and very old patients: a population-based study on incidence. J Am Acad Dermatol 41: 266-268, 1999.

5. Grattan CE: Evidence of an association between bullous pemphigoid and psoriasis. Br J Dermatol 113: 281-283, 1985.

6. Chen KR, Shimizu S, Miyakawa S, Miyakawa S, Ishiko A, Shimizu $\mathrm{H}$ and Hashimoto T: Coexistence of psoriasis and an unusual IgG-mediated subepidermal bullous dermatosis: identification of a novel $200-\mathrm{kDa}$ lower lamina lucida target antigen. Br J Dermatol 134: 340-346, 1996.

7. Wozniak K, Kazama T and Kowalewski C: A practical technique for differentiation of subepidermal bullous diseases: localization of in vivo-bound IgG by laser scanning confocal microscopy. Arch Dermatol 139: 1007-1011, 2003.

8. Bloom D: Bullous eruption in a psoriatic patient. Arch Dermatol 20: 254-255, 1929.

9. Person JR and Rogers RS III: Bullous pemphigoid and psoriasis: does subclinical bullous pemphigoid exist? Br J Dermatol 95: 535-540, 1976.

10. Koerber WA Jr, Price NM and Watson W: Coexistent psoriasis and bullous pemphigoid: a report of six cases. Arch Dermatol 114: 1643-1646, 1978

11. Robinson JK, Baughman RD and Provost TT: Bullous pemphigoid induced by PUVA therapy. Is this the aetiology of the acral bullae produced during PUVA treatment? Br J Dermatol 99: 709-713, 1978 .

12. Abel EA and Bennett A: Bullous pemphigoid. Occurrence in psoriasis treated with psoralens plus long-wave ultraviolet radiation. Arch Dermatol 115: 988-989, 1979.

13. Saeki H, Hayashi N, Komine M, Soma Y, Shimada S, Watanabe $\mathrm{K}$ and Hashimoto T: A case of generalized pustular psoriasis followed by bullous disease: an atypical case of bullous pemphigoid or a novel bullous disease? Br J Dermatol 134: 152-155, 1996.

14. Kirtschig G, Chow ET, Venning VA and Wojnarowska FT: Acquired subepidermal bullous diseases associated with psoriasis: a clinical, immunopathological and immunogenetic study. Br J Dermatol 135: 738-745, 1996.

15. Perl S, Rappersberger K, Fodinger D, Anegg B, Honigsmann H and Ortel B: Bullous pemphigoid induced by PUVA therapy. Dermatology 193: 245-247, 1996.

16. Roeder C and Driesch PV: Psoriatic erythroderma and bullous pemphigoid treated successfully with acitretin and azathioprine. Eur J Dermatol 9: 537-539, 1999.

17. Kawahara Y, Zillikens D, Yancey KB, Marinkovich MP, Nie Z, Hashimoto T and Nishikawa T: Subepidermal blistering disease with autoantibodies against a novel dermal 200-kDa antigen. J Dermatol Sci 23: 93-102, 2000.
18. Morris SD, Mallipeddi R, Oyama N, Gratian MJ, Harman KE, Bhogal BS, Black MM, Eady RA, Hashimoto T and McGrath JA: Psoriasis bullosa acquisita. Clin Exp Dermatol 27: 665-669, 2002.

19. Yasuda H, Tomita Y, Shibaki A and Hashimoto T: Two cases of subepidermal blistering disease with anti-p200 or $180-\mathrm{kD}$ bullous pemphigoid antigen associated with psoriasis. Dermatology 209: 149-155, 2004.

20. Shimanovich I, Hirako Y, Sitaru C, Hashimoto T, Brocker EB, Butt E and Zillikens D: The autoantigen of anti-p200 pemphigoid is an acidic noncollagenous N-linked glycoprotein of the cutaneous basement membrane. J Invest Dermatol 121: 1402$1408,2003$.

21. Glinski W, Jarzabek-Chorzelska M, Pierozynska-Dubowska M, Kuligowski M, Glinska-Ferenz M and Jablonska S: Basement membrane zone as a target for human neutrophil elastase in psoriasis. Arch Dermatol Res 282: 506-511, 1990.

22. Verschoore M, Kowalewski C, Jarzabek Chorzelska M, Bernard BA and Darmon YM: Intraepidermal protein leakage of plasma proteins after type stripping of normal skin and uninvolved psoriatic skin. Br J Dermatol 122: 391-397, 1990.

23. Verraes S, Hornebeck W, Polette M, Borradori L and Bernard P: Respective contribution of neutrophil elastase and matrix metalloproteinase 9 in the degradation of BP180 (type XVII collagen) in human bullous pemphigoid. J Invest Dermatol 117: 1091-1096, 2001.

24. Shimanovich I, Mihai S, Oostingh GJ, Ilenchuk TT, Brocker EB, Opdenakker G, Zillikens D and Sitaru C: Granulocyte-derived elastase and gelatinase B are required for dermal-epidermal separation induced by autoantibodies from patients with epidermolysis bullosa acquisita and bullous pemphigoid. J Pathol 204: 519-527, 2004.

25. Wiedow O, Wiese F, Streit V, Kalm C and Christophers E: Lesional elastase activity in psoriasis, contact dermatitis, and atopic dermatitis. J Invest Dermatol 99: 306-309, 1992.

26. Primka EJ III and Camisa C: Psoriasis and bullous pemphigoid treated with azathioprine. J Am Acad Dermatol 39: 121-123, 1998.

27. Thomsen K and Schmidt H: PUVA-induced bullous pemphigoid. Br J Dermatol 95: 568-569, 1976.

28. Pfau A, Hohenleutner U, Hohenleutner S, Eckert F and Landthaler M: UV-A-provoked localized bullous pemphigoid. Acta Derm Venereol 74: 314-316, 1994.

29. Sacher C, Konig C, Scharffetter-Kochanek K, Krieg T and Hunzelmann N: Bullous pemphigoid in a patient treated with UVA-1 phototherapy for disseminated morphea. Dermatology 202: 54-57, 2001.

30. Preesman AH, Toonstra J, Van der Putte SC, Van Veelden H, De Geer DB, and Van Vloten WA: UV-B-induced bullous pemphigoid restricted to mycosis fungoides plaques. Clin Exp Dermatol 15: 363-366, 1990.

31. Hisler BM, Blumenthal NC, Aronson PJ, Hashimoto K and Rudner EJ: Bullous pemphigoid in psoriatic lesions. J Am Acad Dermatol 20: 683-684, 1989.

32. Ahmed AR and Winkler NW: Psoriasis and bullous pemphigoid. Arch Dermatol 113: 845, 1977.

33. Bianchi L, Gatti S and Nini G: Bullous pemphigoid and severe erythrodermic psoriasis: combined low-dose treatment with cyclosporine and systemic steroids. J Am Acad Dermatol 27: 278,1992 .

34. Boixeda JP, Soria C, Medina S and Ledo A: Bullous pemphigoid and psoriasis: treatment with cyclosporine. J Am Acad Dermatol 24: 152, 1991.

35. Bork K: Psoriasis and bullous pemphigoid. Hautarzt 38: 348351, 1987.

36. Kobayashi TT, Elston DM, Libow LF and David-Bajar K: A case of bullous pemphigoid limited to psoriatic plaques. Cutis 70: 283-287, 2002.

37. Nousari HC, Sragovich A, Kimyai-Asadi A, Orlinsky D and Anhalt GJ: Mycophenolate mofetil in autoimmune and inflammatory skin disorders. J Am Acad Dermatol 40: 265-268, 1999. 